\title{
On two kinds of the reverse half-discrete Mulholland-type inequalities involving higher-order derivative function
}

Qiang Chen ${ }^{1 *}$ and Bicheng Yang ${ }^{2}$

"Correspondence:

cq_c123@163.com

1Department of Computer Science,

Guangdong University of

Education, Guangzhou, Guangdong

510303, P.R. China

Full list of author information is

available at the end of the article

\begin{abstract}
By means of the weight functions, Hermite-Hadamard's inequality, and the techniques of real analysis, a new more accurate reverse half-discrete Mulholland-type inequality involving one higher-order derivative function is given. The equivalent statements of the best possible constant factor related to a few parameters, the equivalent forms, and several particular inequalities are provided. Another kind of the reverses is also considered.

MSC: 26D15; 26D10; 26A42

Keywords: Weight function; Hermite-Hadamard's inequality; Half-discrete Mulholland-type inequality; Higher-order derivative function; Parameter; Best possible constant factor
\end{abstract}

\section{Introduction}

Suppose that $p>1, \frac{1}{p}+\frac{1}{q}=1, a_{m}, b_{n} \geq 0,0<\sum_{m=1}^{\infty} a_{m}^{p}<\infty$, and $0<\sum_{n=1}^{\infty} b_{n}^{q}<\infty$. We have the following Hardy-Hilbert's inequality with the best possible constant factor $\frac{\pi}{\sin (\pi / p)}$ (cf. [1], Theorem 315):

$$
\sum_{m=1}^{\infty} \sum_{n=1}^{\infty} \frac{1}{m+n} a_{m} b_{n}<\frac{\pi}{\sin (\pi / p)}\left(\sum_{m=1}^{\infty} a_{m}^{p}\right)^{\frac{1}{p}}\left(\sum_{n=1}^{\infty} b_{n}^{q}\right)^{\frac{1}{q}} .
$$

Replacing $\frac{1}{m+n}$ with $\frac{1}{m+n-1}$ in (1), we have a more accurate form of (1) (cf. [1], Theorem 323). We still have the following Mulholland's inequality with the same best possible constant factor of (1) (cf. [1], Theorem 343):

$$
\sum_{m=2}^{\infty} \sum_{n=2}^{\infty} \frac{1}{\ln m n} a_{m} b_{n}<\frac{\pi}{\sin (\pi / p)}\left(\sum_{m=2}^{\infty} m^{p-1} a_{m}^{p}\right)^{\frac{1}{p}}\left(\sum_{n=2}^{\infty} n^{q-1} b_{n}^{q}\right)^{\frac{1}{q}} .
$$

(c) The Author(s) 2021. This article is licensed under a Creative Commons Attribution 4.0 International License, which permits use, sharing, adaptation, distribution and reproduction in any medium or format, as long as you give appropriate credit to the original author(s) and the source, provide a link to the Creative Commons licence, and indicate if changes were made. The images or other third party material in this article are included in the article's Creative Commons licence, unless indicated otherwise in a credit line to the material. If material is not included in the article's Creative Commons licence and your intended use is not permitted by statutory regulation or exceeds the permitted use, you will need to obtain permission directly from the copyright holder. To view a copy of this licence, visit http://creativecommons.org/licenses/by/4.0/. 
In 2006, by means of Euler-Maclaurin's summation formula, Krnic et al. [2] provided an extension of (1) as follows:

$$
\sum_{m=1}^{\infty} \sum_{n=1}^{\infty} \frac{a_{m} b_{n}}{(m+n)^{\lambda}}<B\left(\lambda_{1}, \lambda_{2}\right)\left[\sum_{m=1}^{\infty} m^{p\left(1-\lambda_{1}\right)-1} a_{m}^{p}\right]^{\frac{1}{p}}\left[\sum_{n=1}^{\infty} n^{q\left(1-\lambda_{2}\right)-1} b_{n}^{q}\right]^{\frac{1}{q}},
$$

where $\lambda_{i} \in(0,2](i=1,2), \lambda_{1}+\lambda_{2}=\lambda \in(0,4]$, and the constant factor $B\left(\lambda_{1}, \lambda_{2}\right)$ is best possible, $B(u, v)=\int_{0}^{\infty} \frac{t^{u-1}}{(1+t)^{u+v}} d t(u, v>0)$ is the beta function. For $p=q=2, \lambda_{1}=\lambda_{2}=\frac{\lambda}{2}$, inequality (3) reduces to the published result in [3].

In 2019, by using (2) and Abel's summation by parts formula, Adiyasuren et al. [4] published a Hardy-Hilbert-type inequality with the kernel as $\frac{1}{(m+n)^{\lambda}}$ involving partial sums. Inequalities (1)-(3) with their reverses play an important role in analysis and its applications (cf. [5-15]).

In 1934, Hardy et al. [1] also published a half-discrete Hilbert-type inequality in Theorem 351: If $K(t)(t>0)$ is decreasing, $p>1, \frac{1}{p}+\frac{1}{q}=1,0<\phi(s)=\int_{0}^{\infty} K(t) t^{s-1} d t<\infty, a_{n} \geq 0$, such that $0<\sum_{n=1}^{\infty} a_{n}^{p}<\infty$, then

$$
\int_{0}^{\infty} x^{p-2}\left(\sum_{n=1}^{\infty} K(n x) a_{n}\right)^{p} d x<\phi^{p}\left(\frac{1}{q}\right) \sum_{n=1}^{\infty} a_{n}^{p}
$$

Some new extensions of (4) were given in [16-20].

In 2016, by the use of the techniques of real analysis, Hong et al. [21] gave an equivalent statement of the best possible constant factor related to several parameters in the general form of (1). The other similar results were provided by [22-29]. Recently, Yang et al. [30] gave a new result in a reverse half-discrete Hilbert-type inequality.

In this paper, following the way of $[4,21]$, by means of the weight functions, HermiteHadamard's inequality, and the techniques of real analysis, a new more accurate reverse half-discrete Mulholland-type inequality with the kernel as $\frac{1}{\left[x+\ln ^{\alpha}(n-\xi)\right]^{\lambda+m}}$ involving one higher-order derivative function is given. The equivalent statements of the best possible constant factor related to a few parameters, the equivalent forms, and several particular inequalities are provided. Another kind of the reverses is also considered.

\section{Some lemmas}

In what follows, we suppose that $p<1(p \neq 0), \frac{1}{p}+\frac{1}{q}=1, \mathrm{~N}:=\{1,2, \ldots\}, \mathrm{m} \in \mathrm{N} \cup\{0\}$, $\alpha \in(0,1], \xi \in\left[0, \frac{1}{2}\right], \lambda \in(0, \infty), \lambda_{1} \in(0, \lambda), \lambda_{2} \in(0, \lambda) \cap\left(0, \frac{1}{\alpha}\right]$,

$$
k_{\lambda}\left(\lambda_{i}\right):=B\left(\lambda_{i}, \lambda-\lambda_{i}\right) \quad(i=1,2)
$$

$\hat{\lambda}_{1}:=\frac{\lambda-\lambda_{2}}{p}+\frac{\lambda_{1}}{q}, \hat{\lambda}_{2}:=\frac{\lambda-\lambda_{1}}{q}+\frac{\lambda_{2}}{p}$. We also assume that $f(x)=f^{(0)}(x)$ is a continuous derivative function of m-order unless finite points in $R_{+}:=(0, \infty), f^{(k-1)}(y) \geq 0, f^{(k-1)}(0+)=0$, $f^{(k-1)}(x)=o\left(e^{t x}\right)(t>0 ; x \rightarrow \infty)(k=1, \ldots, m)$, and for $f^{(m)}(x), a_{n} \geq 0,0<\int_{0}^{\infty} x^{p\left(1-\hat{\lambda}_{1}\right)-1} \times$ $\left(f^{(m)}(x)\right)^{p} d x<\infty$, and

$$
0<\sum_{n=2}^{\infty}[\ln (n-\xi)]^{q\left(1-\alpha \hat{\lambda}_{2}\right)-1}(n-\xi)^{q-1} a_{n}^{q}<\infty .
$$


Lemma 1 Define the following weight function:

$$
\varpi_{\lambda}\left(\lambda_{2}, x\right):=\alpha x^{\lambda-\lambda_{2}} \sum_{n=2}^{\infty} \frac{[\ln (n-\xi)]^{\alpha \lambda_{2}-1}}{\left[x+\ln ^{\alpha}(n-\xi)\right]^{\lambda}(n-\xi)} \quad\left(x \in \mathrm{R}_{+}\right)
$$

We have the following inequalities:

$$
0<k_{\lambda}\left(\lambda_{2}\right)\left[1-O\left(\frac{1}{x^{\lambda_{2}}}\right)\right]<\varpi_{\lambda}\left(\lambda_{2}, x\right)<k_{\lambda}\left(\lambda_{2}\right) \quad\left(x \in \mathrm{R}_{+}\right),
$$

where we define $O\left(\frac{1}{x^{\lambda} 2}\right):=\frac{1}{k_{\lambda}\left(\lambda_{2}\right)} \int_{0}^{\frac{\ln ^{\alpha}(2-\xi)}{x}} \frac{v^{\lambda_{2}-1}}{(1+\nu)^{\lambda}} d v$ satisfying $0<O\left(\frac{1}{x^{\lambda} 2}\right)<\frac{\ln ^{\alpha \lambda_{2}}(2-\xi)}{k_{\lambda}\left(\lambda_{2}\right) x^{\lambda_{2}}}$.

Proof For fixed $x \in \mathrm{R}_{+}$, the function $g(t):=\frac{[\ln (t-\xi)]^{\alpha \lambda_{2}-1}}{\left[x+\ln ^{\alpha}(t-\xi)\right]^{\lambda}(t-\xi)}$ is strictly decreasing and strictly convex in $\left(\frac{3}{2}, \infty\right)$. In fact, for $\alpha \in(0,1], \lambda_{2} \in\left(0, \frac{1}{\alpha}\right], \xi \in\left[0, \frac{1}{2}\right], t \in\left(\frac{3}{2}, \infty\right)$,

$$
\begin{aligned}
\frac{d}{d \mathrm{t}} g(t)= & -\frac{\left(1-\alpha \lambda_{2}\right)[\ln (t-\xi)]^{\alpha \lambda_{2}-2}}{\left[x+\ln ^{\alpha}(t-\xi)\right]^{\lambda}(t-\xi)^{2}}-\frac{\lambda \alpha[\ln (t-\xi)]^{\alpha \lambda_{2}+\alpha-2}}{\left[x+\ln ^{\alpha}(t-\xi)\right]^{\lambda+1}(t-\xi)^{2}} \\
& -\frac{[\ln (t-\xi)]^{\alpha \lambda_{2}-1}}{\left[x+\ln ^{\alpha}(t-\xi)\right]^{\lambda}(t-\xi)^{2}}<0, \\
\frac{d^{2}}{d \mathrm{t}^{2}} g(t)= & \frac{\left(1-\alpha \lambda_{2}\right)\left(2-\alpha \lambda_{2}\right)[\ln (t-\xi)]^{\alpha \lambda_{2}-3}}{\left[x+\ln ^{\alpha}(t-\xi)\right]^{\lambda}(t-\xi)^{3}}+\frac{\lambda \alpha\left(3-2 \alpha \lambda_{2}-\alpha\right)[\ln (t-\xi)]^{\alpha \lambda_{2}+\alpha-3}}{\left[x+\ln ^{\alpha}(t-\xi)\right]^{\lambda+1}(t-\xi)^{3}} \\
& +\frac{3\left(1-\alpha \lambda_{2}\right)[\ln (t-\xi)]^{\alpha \lambda_{2}-2}}{\left[x+\ln { }^{\alpha}(t-\xi)\right]^{\lambda}(t-\xi)^{3}}+\frac{\lambda \alpha^{2}(\lambda+1)[\ln (t-\xi)]^{\alpha \lambda_{2}+2 \alpha-3}}{[x+\ln (t-\xi)]^{\lambda+2}(t-\xi)^{3}} \\
& +\frac{3 \lambda \alpha[\ln (t-\xi)]^{\alpha \lambda_{2}+\alpha-2}}{\left[x+\ln ^{\alpha}(t-\xi)\right]^{\lambda+1}(t-\xi)^{3}}+\frac{2[\ln (t-\xi)]^{\alpha \lambda_{2}-1}}{\left[x+\ln ^{\alpha}(t-\xi)\right]^{\lambda}(t-\xi)^{3}}>0 .
\end{aligned}
$$

By the decreasingness property of series and Hermite-Hadamard's inequality (cf. [31]), we have

$$
\begin{aligned}
\int_{2}^{\infty} \frac{[\ln (t-\xi)]^{\alpha \lambda_{2}-1}}{\left[x+\ln ^{\alpha}(t-\xi)\right]^{\lambda}(t-\xi)} d t & <\sum_{n=2}^{\infty} \frac{[\ln (n-\xi)]^{\alpha \lambda_{2}-1}}{\left[x+\ln ^{\alpha}(n-\xi)\right]^{\lambda}(n-\xi)} \\
& <\int_{\frac{3}{2}}^{\infty} \frac{[\ln (t-\xi)]^{\alpha \lambda_{2}-1}}{\left[x+\ln ^{\alpha}(t-\xi)\right]^{\lambda}(t-\xi)} d t
\end{aligned}
$$

Setting $v=\frac{\ln ^{\alpha}(t-\xi)}{x}\left(\frac{1}{t-\xi} d t=\frac{1}{\alpha} x^{\frac{1}{\alpha}} V^{\frac{1}{\alpha}-1} d v\right)$, for $\frac{3}{2}-\xi \geq 1$, we obtain

$$
\begin{aligned}
& \int_{\frac{3}{2}}^{\infty} \frac{[\ln (t-\xi)]^{\alpha \lambda_{2}-1}}{\left[x+\ln ^{\alpha}(t-\xi)\right]^{\lambda}(t-\xi)} d t \\
& \quad=\frac{1}{\alpha x^{\lambda}} \int_{\frac{\ln \alpha\left(\frac{3}{2}-\xi\right)}{x}}^{\infty} \frac{(x v)^{\frac{1}{\alpha}\left(\alpha \lambda_{2}-1\right)}}{(1+v)^{\lambda}} x^{\frac{1}{\alpha}} v^{\frac{1}{\alpha}-1} d v \leq \frac{1}{\alpha x^{\lambda-\lambda_{2}}} \int_{0}^{\infty} \frac{v^{\lambda_{2}-1} d v}{(1+v)^{\lambda}}=\frac{1}{\alpha x^{\lambda-\lambda_{2}}} k_{\lambda}\left(\lambda_{2}\right) .
\end{aligned}
$$

By (5) and (7), we have

$$
\varpi_{\lambda}\left(\lambda_{2}, x\right)<\alpha x^{\lambda-\lambda_{2}} \frac{1}{\alpha x^{\lambda-\lambda_{2}}} k_{\lambda}\left(\lambda_{2}\right)=k_{\lambda}\left(\lambda_{2}\right) .
$$


On the other hand, in the same way, we find

$$
\begin{aligned}
\varpi_{\lambda}\left(\lambda_{2}, x\right) & >\alpha x^{\lambda-\lambda_{2}} \int_{2}^{\infty} \frac{[\ln (t-\xi)]^{\alpha \lambda_{2}-1}}{\left[x+\ln ^{\alpha}(t-\xi)\right]^{\lambda}(t-\xi)} d t \\
& =\int_{0}^{\infty} \frac{v^{\lambda_{2}-1} d v}{(1+v)^{\lambda}}-\int_{0}^{\frac{\ln ^{\alpha}(2-\xi)}{x}} \frac{v^{\lambda_{2}-1} d v}{(1+v)^{\lambda}}=k_{\lambda}\left(\lambda_{2}\right)\left[1-O\left(\frac{1}{x^{\lambda_{2}}}\right)\right],
\end{aligned}
$$

where $O\left(\frac{1}{x^{\lambda} 2}\right)=\frac{1}{k_{\lambda}\left(\lambda_{2}\right)} \int_{0}^{\frac{\ln ^{\alpha}(2-\xi)}{x}} \frac{v^{\lambda 2^{-1}}}{(1+v)^{\lambda}} d \nu$ satisfying

$$
0<O\left(\frac{1}{x^{\lambda_{2}}}\right)<\frac{1}{k_{\lambda}\left(\lambda_{2}\right)} \int_{0}^{\frac{\ln ^{\alpha}(2-\xi)}{x}} v^{\lambda_{2}-1} d v=\frac{\ln ^{\alpha \lambda_{2}}(2-\xi)}{k_{\lambda}\left(\lambda_{2}\right) x^{\lambda_{2}}} \quad(x>0) .
$$

Hence, inequalities (6) follow.

The lemma is proved.

Lemma 2 For $p<0(0<q<1)$, we have the following reverse Mulholland-type inequality:

$$
\begin{aligned}
I_{0}:= & \int_{0}^{\infty} \sum_{n=2}^{\infty} \frac{a_{n}}{\left[x+\ln ^{\alpha}(n-\xi)\right]^{\lambda}} f^{(m)}(x) d x>\left(\frac{1}{\alpha} k_{\lambda}\left(\lambda_{2}\right)\right)^{\frac{1}{p}}\left(k_{\lambda}\left(\lambda_{1}\right)\right)^{\frac{1}{q}} \\
& \times\left[\int_{0}^{\infty} x^{p\left(1-\hat{\lambda}_{1}\right)-1}\left(f^{(m)}(x)\right)^{p} d x\right]^{\frac{1}{p}}\left\{\sum_{n=2}^{\infty}[\ln (n-\xi)]^{q\left(1-\alpha \hat{\lambda}_{2}\right)-1}(n-\xi)^{q-1} a_{n}^{q}\right\}^{\frac{1}{q}} .
\end{aligned}
$$

Proof For $\alpha>0$, setting $v=x / \ln ^{\alpha}(n-\xi)$, we can obtain another weight function:

$$
\begin{aligned}
\omega_{\lambda}\left(\lambda_{1}, n\right) & :=[\ln (n-\xi)]^{\alpha\left(\lambda-\lambda_{1}\right)} \int_{0}^{\infty} \frac{x^{\lambda_{1}-1} d x}{\left[x+\ln ^{\alpha}(n-\xi)\right]^{\lambda}} \\
& =\int_{0}^{\infty} \frac{v^{\lambda_{1}-1} d v}{(v+1)^{\lambda}}=k_{\lambda}\left(\lambda_{1}\right) \quad(n \in \mathrm{N} \backslash\{1\}) .
\end{aligned}
$$

By reverse Hölder's inequality (cf. [31]), we have

$$
\begin{aligned}
I_{0}= & \int_{0}^{\infty} \sum_{n=2}^{\infty} \frac{1}{\left[x+\ln ^{\alpha}(n-\xi)\right]^{\lambda}}\left\{\frac{x^{\left(1-\lambda_{1}\right) / q}(n-\xi)^{-1 / p}}{[\ln (n-\xi)]^{\left(1-\alpha \lambda_{2}\right) / p}} f^{(m)}(x)\right\}\left\{\frac{[\ln (n-\xi)]^{\left(1-\alpha \lambda_{2}\right) / p}}{x^{\left(1-\lambda_{1}\right) / q(n-\xi)^{-1 / p}}} a_{n}\right\} d x \\
\geq & \left\{\int_{0}^{\infty} \sum_{n=2}^{\infty} \frac{1}{\left[x+\ln ^{\alpha}(n-\xi)\right]^{\lambda}} \frac{x^{\left(1-\lambda_{1}\right)(p-1)}(n-\xi)^{-1}}{[\ln (n-\xi)]^{1-\alpha \lambda_{2}}}\left(f^{(m)}(x)\right)^{p} d x\right\}^{\frac{1}{p}} \\
& \times\left\{\sum_{n=2}^{\infty} \int_{0}^{\infty} \frac{1}{\left[x+\ln ^{\alpha}(n-\xi)\right]^{\lambda}} \frac{[\ln (n-\xi)]^{\left(1-\alpha \lambda_{2}\right)(q-1)} a_{n}^{q}}{x^{1-\lambda_{1}}(n-\xi)^{1-q}} d x\right\}^{\frac{1}{q}} \\
= & \left\{\frac{1}{\alpha} \int_{0}^{\infty} \varpi_{\lambda}\left(\lambda_{2}, x\right) x^{p\left(1-\hat{\lambda}_{1}\right)-1}\left(f^{(m)}(x)\right)^{p} d x\right\}^{\frac{1}{p}} \\
& \times\left\{\sum_{n=2}^{\infty} \omega_{\lambda}\left(\lambda_{1}, n\right)[\ln (n-\xi)]^{q\left(1-\alpha \hat{\lambda}_{2}\right)-1}(n-\xi)^{q-1} a_{n}^{q}\right\}^{\frac{1}{q}} .
\end{aligned}
$$


We show that (10) does not keep the form of equality. Otherwise (cf. [31]), there exist constants $A$ and $B$ such that both of them are not zero and

$$
A \frac{x^{\left(1-\lambda_{1}\right)(p-1)}(n-\xi)^{-1}}{[\ln (n-\xi)]^{1-\alpha \lambda_{2}}}\left(f^{(m)}(x)\right)^{p}=B \frac{[\ln (n-\xi)]^{\left(1-\alpha \lambda_{2}\right)(q-1)} a_{n}^{q}}{x^{1-\lambda_{1}}(n-\xi)^{1-q}} \quad \text { a.e. in } \mathrm{R}_{+} \times \mathrm{N} \backslash\{1\}
$$

Assuming that $A \neq 0$, there exists $n \in \mathrm{N} \backslash\{1\}$ satisfying

$$
x^{p\left(1-\hat{\lambda}_{1}\right)-1}\left(f^{(m)}(x)\right)^{p}=\frac{B}{A}[\ln (n-\xi)]^{q\left(1-\alpha \lambda_{2}\right)}(n-\xi)^{-q} a_{n}^{q} \frac{1}{x^{1+\left(\lambda-\lambda_{1}-\lambda_{2}\right)}} \quad \text { a.e. in } \mathrm{R}_{+},
$$

which contradicts the fact that $0<\int_{0}^{\infty} x^{p\left(1-\hat{\lambda}_{1}\right)-1}\left(f^{(m)}(x)\right)^{p} d x<\infty$, since $\int_{0}^{\infty} \frac{1}{x^{1+\left(\lambda-\lambda_{1}-\lambda_{2}\right)}} d x=$ $\infty$. Then, by (6) and (9), we have (8).

The lemma is proved.

Lemma 3 For $t>0$, we have the following expression:

$$
\int_{0}^{\infty} e^{-t x} f(x) d x=\frac{1}{t^{m}} \int_{0}^{\infty} e^{-t x} f^{(m)}(x) d x
$$

Proof For $m=0$, in view of $f^{(0)}(x)=f(x),(11)$ is valid. For $m \in \mathrm{N}$, since $f^{(k-1)}(0+)=0(k=$ $1, \ldots, m)$, integration by parts, we find

$$
\begin{aligned}
\int_{0}^{\infty} e^{-t x} f^{(k)}(x) d x & =\int_{0}^{\infty} e^{-t x} d f^{(k-1)}(x) \\
& =\left.e^{-t x} f^{(k-1)}(x)\right|_{0} ^{\infty}-\int_{0}^{\infty} f^{(k-1)}(x) d e^{-t x} \\
& =\lim _{x \rightarrow \infty} \frac{f^{(k-1)}(x)}{e^{t x}}+t \int_{0}^{\infty} e^{-t x} f^{(k-1)}(x) d x
\end{aligned}
$$

In view of

$$
f^{(k-1)}(x)=o\left(e^{t x}\right) \quad(t>0, x \rightarrow \infty ; k=1, \ldots, m),
$$

it follows that $\lim _{x \rightarrow \infty} \frac{f^{(k-1)}(x)}{e^{t x}}=0$, and then

$$
\int_{0}^{\infty} e^{-t x} f^{(k-1)}(x) d x=\frac{1}{t} \int_{0}^{\infty} e^{-t x} f^{(k)}(x) d x .
$$

By substitution of $k=1, \ldots, m$ in the above expression, (11) follows.

The lemma is proved.

\section{Main results}

Theorem 1 For $p<0(0<q<1)$, we have the following more accurate reverse half-discrete Mulholland-type inequality involving one higher-order derivative function:

$$
I:=\int_{0}^{\infty} \sum_{n=2}^{\infty} \frac{a_{n}}{\left[x+\ln ^{\alpha}(n-\xi)\right]^{\lambda+m}} f(x) d x>\frac{\Gamma(\lambda)}{\Gamma(\lambda+m)}\left(\frac{1}{\alpha} k_{\lambda}\left(\lambda_{2}\right)\right)^{\frac{1}{p}}\left(k_{\lambda}\left(\lambda_{1}\right)\right)^{\frac{1}{q}}
$$




$$
\times\left[\int_{0}^{\infty} x^{p\left(1-\overleftarrow{\lambda_{1}}\right)-1}\left(f^{(m)}(x)\right)^{p} d x\right]^{\frac{1}{p}}\left\{\sum_{n=2}^{\infty}[\ln (n-\xi)]^{q\left(1-\alpha \overleftarrow{\lambda_{2}}\right)-1}(n-\xi)^{q-1} a_{n}^{q}\right\}^{\frac{1}{q}}
$$

In particular, for $\lambda_{1}+\lambda_{2}=\lambda$, we have

$$
0<\int_{0}^{\infty} x^{p\left(1-\lambda_{1}\right)-1}\left(f^{(m)}(x)\right)^{p} d x<\infty, \quad 0<\sum_{n=2}^{\infty}[\ln (n-\xi)]^{q\left(1-\alpha \lambda_{2}\right)-1}(n-\xi)^{q-1} a_{n}^{q}<\infty,
$$

and the following inequality:

$$
\begin{aligned}
I= & \int_{0}^{\infty} \sum_{n=2}^{\infty} \frac{a_{n}}{\left[x+\ln ^{\alpha}(n-\xi)\right]^{\lambda+m}} f(x) d x \\
> & \frac{\Gamma(\lambda)}{\alpha^{1 / p} \Gamma(\lambda+m)} B\left(\lambda_{1}, \lambda_{2}\right) \\
& \times\left[\int_{0}^{\infty} x^{p\left(1-\lambda_{1}\right)-1}\left(f^{(m)}(x)\right)^{p} d x\right]^{\frac{1}{p}}\left\{\sum_{n=2}^{\infty}[\ln (n-\xi)]^{q\left(1-\alpha \lambda_{2}\right)-1}(n-\xi)^{q-1} a_{n}^{q}\right\}^{\frac{1}{q}} .
\end{aligned}
$$

Proof Since we have

$$
\frac{1}{\left[x+\ln ^{\alpha}(n-\xi)\right]^{\lambda+m}}=\frac{1}{\Gamma(\lambda+m)} \int_{0}^{\infty} t^{\lambda+m-1} e^{-\left[x+\ln ^{\alpha}(n-\xi)\right] t} d t,
$$

by the Lebesgue term by term integration theorem (cf. [32]) and (11), we find

$$
\begin{aligned}
I & =\frac{1}{\Gamma(\lambda+m)} \int_{0}^{\infty} \sum_{n=2}^{\infty} a_{n} f(x) \int_{0}^{\infty} t^{\lambda+m-1} e^{-\left[x+\ln ^{\alpha}(n-\xi)\right] t} d t d x \\
& =\frac{1}{\Gamma(\lambda+m)} \int_{0}^{\infty} t^{\lambda+m-1}\left(\int_{0}^{\infty} e^{-x t} f(x) d x\right) \sum_{n=2}^{\infty} e^{-t \ln ^{\alpha}(n-\xi)} a_{n} d t \\
& =\frac{1}{\Gamma(\lambda+m)} \int_{0}^{\infty} t^{\lambda+m-1}\left(t^{-m} \int_{0}^{\infty} e^{-x t} f^{(m)}(x) d x\right) \sum_{n=2}^{\infty} e^{-t \ln ^{\alpha}(n-\xi)} a_{n} d t \\
& =\frac{1}{\Gamma(\lambda+m)} \int_{0}^{\infty} \sum_{n=2}^{\infty} a_{n} f^{(m)}(x)\left\{\int_{0}^{\infty} t^{\lambda-1} e^{-\left[x+\ln ^{\alpha}(n-\xi)\right] t} d t\right\} d x \\
& =\frac{\Gamma(\lambda)}{\Gamma(\lambda+m)} \int_{0}^{\infty} \sum_{n=2}^{\infty} \frac{a_{n}}{\left[x+\ln ^{\alpha}(n-\xi)\right]^{\lambda}} f^{(m)}(x) d x=\frac{\Gamma(\lambda)}{\Gamma(\lambda+m)} I_{0} .
\end{aligned}
$$

Then, by (8), we have (12).

The theorem is proved.

Theorem 2 For $p<0(0<q<1)$, if $\lambda_{1}+\lambda_{2}=\lambda$, then the constant factor

$$
\frac{\Gamma(\lambda)}{\Gamma(\lambda+m)}\left(\frac{1}{\alpha} k_{\lambda}\left(\lambda_{2}\right)\right)^{\frac{1}{p}}\left(k_{\lambda}\left(\lambda_{1}\right)\right)^{\frac{1}{q}}
$$

in (12) is best possible. On the other hand, if we add the condition that $\lambda-\lambda_{1} \leq \frac{1}{\alpha}$, the same constant factor in (12) is best possible, then $\lambda_{1}+\lambda_{2}=\lambda$. 
Proof In the following, for $m=0, \varepsilon \geq 0$, we define $\prod_{i=0}^{m-1}\left(\lambda_{1}+i-\frac{\varepsilon}{p}\right)=1$.

If $\lambda_{1}+\lambda_{2}=\lambda$, then (12) reduces to (13). For any $0<\varepsilon<q \lambda_{2}$, we set

$$
\begin{aligned}
& \tilde{f}^{(0)}(x)=\tilde{f}(x):=\left\{\begin{array}{ll}
0, & 0<x<1, \\
x^{\lambda_{1}+m-\frac{\varepsilon}{p}-1}, & x \geq 1
\end{array},\right. \\
& \tilde{a}_{n}:=[\ln (n-\xi)]^{\alpha\left(\lambda_{2}-\frac{\varepsilon}{q}\right)-1}(n-\xi)^{-1} \quad(n \in \mathrm{N} \backslash\{1\}), .
\end{aligned}
$$

and find

$$
\tilde{f}^{(k)}(x)=\left\{\begin{array}{l}
0, \quad 0<x<1, \\
\prod_{i=0}^{k-1}\left(\lambda_{1}+i-\frac{\varepsilon}{p}\right) x^{\lambda_{1}+m-k-\frac{\varepsilon}{p}-1}, \quad x>1
\end{array} \quad(k=0, \ldots, m)\right.
$$

satisfying $\tilde{f}^{(k)}(0+)=0, \tilde{f}^{(k)}(x)=o\left(e^{t x}\right)(t>0, x \rightarrow \infty ; k=0, \ldots, m-1)$.

If there exists a constant $M\left(\geq \frac{\Gamma(\lambda)}{\alpha^{1 / p} \Gamma(\lambda+m)} B\left(\lambda_{1}, \lambda_{2}\right)\right)$ such that (13) is valid when we replace $\frac{\Gamma(\lambda)}{\alpha^{1 / p} \Gamma(\lambda+m)} B\left(\lambda_{1}, \lambda_{2}\right)$ with $M$, then in particular we have

$$
\begin{aligned}
\tilde{I} & :=\int_{0}^{\infty} \sum_{n=2}^{\infty} \frac{\tilde{a}_{n} \tilde{f}(x)}{\left[x+\ln ^{\alpha}(n-\xi)\right]^{\lambda+m}} d x \\
& >M\left[\int_{0}^{\infty} x^{p\left(1-\lambda_{1}\right)-1}\left(\tilde{f}^{(m)}(x)\right)^{p} d x\right]^{\frac{1}{p}}\left\{\sum_{n=2}^{\infty}[\ln (n-\xi)]^{q\left(1-\alpha \lambda_{2}\right)-1}(n-\xi)^{q-1} \tilde{a}_{n}^{q}\right\}^{\frac{1}{q}} .
\end{aligned}
$$

By the decreasingness property of series, we find

$$
\begin{aligned}
\tilde{I}> & M \prod_{i=0}^{m-1}\left(\lambda_{1}+i-\frac{\varepsilon}{p}\right)\left[\int_{1}^{\infty} x^{p\left(1-\lambda_{1}\right)-1} x^{p\left(\lambda_{1}-1\right)-\varepsilon} d x\right]^{\frac{1}{p}} \\
& \times\left\{\sum_{n=2}^{\infty}[\ln (n-\xi)]^{q\left(1-\alpha \lambda_{2}\right)-1}(n-\xi)^{q-1}[\ln (n-\xi)]^{q \alpha \lambda_{2}-\alpha \varepsilon-q}(n-\xi)^{-q}\right\}^{\frac{1}{q}} \\
= & M \prod_{i=0}^{m-1}\left(\lambda_{1}+i-\frac{\varepsilon}{p}\right)\left(\int_{1}^{\infty} x^{-\varepsilon-1} d x\right)^{\frac{1}{p}}\left\{\sum_{n=2}^{\infty}[\ln (n-\xi)]^{-\alpha \varepsilon-1}(n-\xi)^{-1}\right\}^{\frac{1}{q}} \\
> & M \prod_{i=0}^{m-1}\left(\lambda_{1}+i-\frac{\varepsilon}{p}\right)\left(\int_{1}^{\infty} x^{-\varepsilon-1} d x\right)^{\frac{1}{p}}\left\{\int_{2}^{\infty}[\ln (y-\xi)]^{-\alpha \varepsilon-1}(y-\xi)^{-1} d y\right\}^{\frac{1}{q}} \\
= & \frac{M}{\varepsilon} \prod_{i=0}^{m-1}\left(\lambda_{1}+i-\frac{\varepsilon}{p}\right)\left\{\frac{1}{\alpha}[\ln (2-\xi)]^{-\alpha \varepsilon}\right\}^{\frac{1}{q}} .
\end{aligned}
$$

Replacing $\lambda$ with $\lambda+m$, setting $\tilde{\lambda}_{2}:=\lambda_{2}-\frac{\varepsilon}{q} \in(0, \lambda+m) \cap\left(0, \frac{1}{\alpha}\right], \tilde{\lambda}_{1}:=\lambda_{1}+m+\frac{\varepsilon}{q} \in(0, \lambda+m)$ in (5), by (6), we have

$$
\tilde{I}=\int_{1}^{\infty}\left\{x^{\lambda_{1}+m+\frac{\varepsilon}{q}} \sum_{n=2}^{\infty} \frac{[\ln (n-\xi)]^{\alpha\left(\lambda_{2}-\frac{\varepsilon}{q}\right)-1}}{\left[x+\ln ^{\alpha}(n-\xi)\right]^{\lambda+m}(n-\xi)}\right\} x^{-\varepsilon-1} d x
$$




$$
\begin{aligned}
& =\frac{1}{\alpha} \int_{1}^{\infty} \varpi_{\lambda+m}\left(\tilde{\lambda}_{2}, x\right) x^{-\varepsilon-1} d x \\
& <\frac{1}{\alpha} \int_{1}^{\infty} k_{\lambda+m}\left(\tilde{\lambda}_{2}\right) x^{-\varepsilon-1} d x=\frac{1}{\varepsilon \alpha} k_{\lambda+m}\left(\tilde{\lambda}_{2}\right) .
\end{aligned}
$$

Based on the above results, we have

$$
\frac{1}{\alpha} k_{\lambda+m}\left(\tilde{\lambda}_{2}\right)>\varepsilon \tilde{I}>M \prod_{i=0}^{m-1}\left(\lambda_{1}+i-\frac{\varepsilon}{p}\right)\left\{\frac{1}{\alpha}[\ln (2-\xi)]^{-\alpha \varepsilon}\right\}^{\frac{1}{q}} .
$$

For $\varepsilon \rightarrow 0^{+}$, in view of the continuity of the beta function, it follows that

$$
\frac{\Gamma(\lambda)}{\alpha^{1 / p} \Gamma(\lambda+m)} B\left(\lambda_{1}, \lambda_{2}\right)=\frac{\Gamma\left(\lambda_{1}\right) \Gamma\left(\lambda_{2}\right)}{\alpha^{1 / p} \Gamma(\lambda+m)}=\frac{B\left(\lambda_{1}+m, \lambda_{2}\right)}{\alpha^{1 / p} \prod_{i=0}^{m-1}\left(\lambda_{1}+i\right)} \geq M
$$

Hence, $M=\frac{\Gamma(\lambda)}{\alpha^{1 / p} \Gamma(\lambda+m)} B\left(\lambda_{1}, \lambda_{2}\right)$ is the best possible constant factor in (13).

On the other hand, for $\hat{\lambda}_{1}=\frac{\lambda-\lambda_{2}}{p}+\frac{\lambda_{1}}{q}, \hat{\lambda}_{2}=\frac{\lambda-\lambda_{1}}{q}+\frac{\lambda_{2}}{p}, \lambda-\lambda_{1} \leq \frac{1}{\alpha}$, we find

$$
\begin{aligned}
& \hat{\lambda}_{1}+\hat{\lambda}_{2}=\frac{\lambda-\lambda_{2}}{p}+\frac{\lambda_{1}}{q}+\frac{\lambda-\lambda_{1}}{q}+\frac{\lambda_{2}}{p}=\lambda, \\
& 0<\hat{\lambda}_{1}, \quad \hat{\lambda}_{2}<\frac{\lambda}{p}+\frac{\lambda}{q}=\lambda, \quad \hat{\lambda}_{2} \leq \frac{1 / \alpha}{p}+\frac{1 / \alpha}{q}=\frac{1}{\alpha},
\end{aligned}
$$

and $\frac{\Gamma(\lambda)}{\alpha^{1 / p} \Gamma(\lambda+m)} B\left(\hat{\lambda}_{1}, \hat{\lambda}_{2}\right) \in \mathrm{R}_{+}$. Substituting $\hat{\lambda}_{i}=\lambda_{i}(i=1,2)$ in (13), we still have

$$
\begin{aligned}
I= & \int_{0}^{\infty} \sum_{n=2}^{\infty} \frac{a_{n}}{\left[x+\ln ^{\alpha}(n-\xi)\right]^{\lambda+m}} f(x) d x \\
> & \frac{\Gamma(\lambda)}{\alpha^{1 / p} \Gamma(\lambda+m)} B\left(\hat{\lambda}_{1}, \hat{\lambda}_{2}\right) \\
& \times\left[\int_{0}^{\infty} x^{p\left(1-\hat{\lambda}_{1}\right)-1}\left(f^{(m)}(x)\right)^{p} d x\right]^{\frac{1}{p}}\left\{\sum_{n=2}^{\infty}[\ln (n-\xi)]^{q\left(1-\alpha \hat{\lambda}_{2}\right)-1}(n-\xi)^{q-1} a_{n}^{q}\right\}^{\frac{1}{q}} .
\end{aligned}
$$

By reverse Hölder's inequality (cf. [31]), we still have

$$
\begin{aligned}
B\left(\hat{\lambda}_{1}, \hat{\lambda}_{2}\right)= & k_{\lambda}\left(\frac{\lambda-\lambda_{2}}{p}+\frac{\lambda_{1}}{q}\right) \\
= & \int_{0}^{\infty} \frac{1}{(1+u)^{\lambda}} u^{\frac{\lambda-\lambda_{2}}{p}+\frac{\lambda_{1}}{q}-1} d u=\int_{0}^{\infty} \frac{1}{(1+u)^{\lambda}}\left(u^{\frac{\lambda-\lambda_{2}-1}{p}}\right)\left(u^{\frac{\lambda_{1}-1}{q}}\right) d u \\
\geq & {\left[\int_{0}^{\infty} \frac{1}{(1+u)^{\lambda}} u^{\lambda-\lambda_{2}-1} d u\right]^{\frac{1}{p}}\left[\int_{0}^{\infty} \frac{1}{(1+u)^{\lambda}} u^{\lambda_{1}-1} d u\right]^{\frac{1}{q}} } \\
= & {\left[\int_{0}^{\infty} \frac{1}{(1+v)^{\lambda}} v^{\lambda_{2}-1} d v\right]^{\frac{1}{p}}\left[\int_{0}^{\infty} \frac{1}{(1+u)^{\lambda}} u^{\lambda_{1}-1} d u\right]^{\frac{1}{q}} } \\
& =\left(k_{\lambda}\left(\lambda_{2}\right)\right)^{\frac{1}{p}}\left(k_{\lambda}\left(\lambda_{1}\right)\right)^{\frac{1}{q}} .
\end{aligned}
$$


In view of $\frac{\Gamma(\lambda)}{\alpha^{1 / p} \Gamma(\lambda+m)}\left(k_{\lambda}\left(\lambda_{2}\right)\right)^{\frac{1}{p}}\left(k_{\lambda}\left(\lambda_{1}\right)\right)^{\frac{1}{q}}$ being the best possible constant factor in (12), by (15), we have the following inequality:

$$
\frac{\Gamma(\lambda)}{\alpha^{1 / p} \Gamma(\lambda+m)}\left(k_{\lambda}\left(\lambda_{2}\right)\right)^{\frac{1}{p}}\left(k_{\lambda}\left(\lambda_{1}\right)\right)^{\frac{1}{q}} \geq \frac{\Gamma(\lambda)}{\alpha^{1 \cdot p} \Gamma(\lambda+m)} B\left(\hat{\lambda}_{1}, \hat{\lambda}_{2}\right)\left(\in \mathrm{R}_{+}\right),
$$

namely, $B\left(\hat{\lambda}_{1}, \hat{\lambda}_{2}\right) \leq\left(k_{\lambda}\left(\lambda_{2}\right)\right)^{\frac{1}{p}}\left(k_{\lambda}\left(\lambda_{1}\right)\right)^{\frac{1}{q}}$, and then (16) keeps the form of equality.

We observe that (16) keeps the form of equality if and only if there exist constants $A$ and $B$ (cf. [31]) such that they are not both zero and $A u^{\lambda-\lambda_{2}}=B u^{\lambda_{1}}$ a.e. in $\mathrm{R}_{+}$. Assuming that $A \neq 0$, we find $u^{\lambda-\lambda_{2}-\lambda_{1}}=\frac{B}{A}$ a.e. in $\mathrm{R}_{+}$, namely, $\lambda-\lambda_{2}-\lambda_{1}=0$. Hence we have $\lambda_{1}+\lambda_{2}=\lambda$.

The theorem is proved.

\section{Equivalent forms and some particular inequalities}

Theorem 3 For $p<0(0<q<1)$, we have the following reverse half-discrete Mulhollandtype inequality equivalent to (12):

$$
\begin{aligned}
J & :=\left\{\sum_{n=2}^{\infty}[\ln (n-\xi)]^{p \alpha \hat{\lambda}_{2}-1}(n-\xi)^{-1}\left[\int_{0}^{\infty} \frac{f(x)}{\left[x+\ln ^{\alpha}(n-\xi)\right]^{\lambda+m}} d x\right]^{p}\right\}^{\frac{1}{p}} \\
& >\frac{\Gamma(\lambda)}{\alpha^{1 / p} \Gamma(\lambda+m)}\left(k_{\lambda}\left(\lambda_{2}\right)\right)^{\frac{1}{p}}\left(k_{\lambda}\left(\lambda_{1}\right)\right)^{\frac{1}{q}}\left[\int_{0}^{\infty} x^{p\left(1-\hat{\lambda}_{1}\right)-1}\left(f^{(m)}(x)\right)^{p} d x\right]^{\frac{1}{p}} .
\end{aligned}
$$

In particular, for $\lambda_{1}+\lambda_{2}=\lambda$, we have the following inequality equivalent to (13):

$$
\begin{gathered}
\left\{\sum_{n=2}^{\infty}[\ln (n-\xi)]^{p \alpha \lambda_{2}-1}(n-\xi)^{-1}\left[\int_{0}^{\infty} \frac{f(x)}{\left[x+\ln ^{\alpha}(n-\xi)\right]^{\lambda+m}} d x\right]^{p}\right\}^{\frac{1}{p}} \\
>\frac{\Gamma(\lambda)}{\alpha^{1 / p} \Gamma(\lambda+m)} B\left(\lambda_{1}, \lambda_{2}\right)\left[\int_{0}^{\infty} x^{p\left(1-\lambda_{1}\right)-1}\left(f^{(m)}(x)\right)^{p} d x\right]^{\frac{1}{p}} .
\end{gathered}
$$

Proof Suppose that (17) is valid. By reverse Hölder's inequality, we have

$$
\begin{aligned}
I= & \sum_{n=2}^{\infty}\left\{[\ln (n-\xi)]^{\alpha \hat{\lambda}_{2}-\frac{1}{p}}(n-\xi)^{-\frac{1}{p}} \int_{0}^{\infty} \frac{f(x) d x}{\left[x+\ln ^{\alpha}(n-\xi)\right]^{\lambda+m}}\right\} \\
& \times\left\{[\ln (n-\xi)]^{-\alpha \hat{\lambda}_{2}+\frac{1}{p}}(n-\xi)^{\frac{1}{p}} a_{n}\right\} \\
\geq & J\left\{\sum_{n=2}^{\infty}[\ln (n-\xi)]^{q\left(1-\alpha \hat{\lambda}_{2}\right)-1}(n-\xi)^{q-1} a_{n}^{q}\right\}^{\frac{1}{q}} .
\end{aligned}
$$

Then, by (17), we have (12).

On the other hand, assuming that (12) is valid, we set

$$
a_{n}:=[\ln (n-\xi)]^{p \alpha \lambda_{2}-1}(n-\xi)^{-1}\left[\int_{0}^{\infty} \frac{f(x)}{\left[x+\ln ^{\alpha}(n-\xi)\right]^{\lambda+m}} d x\right]^{p-1}, \quad n \in \mathrm{N} \backslash\{1\}
$$


If $J=\infty$, then (17) is naturally valid; if $J=0$, then it is impossible that makes (17) valid, i. e., $J>0$. Suppose that $0<J<\infty$. By (12), we have

$$
\begin{aligned}
\infty & >\sum_{n=2}^{\infty}[\ln (n-\xi)]^{q\left(1-\alpha \hat{\lambda}_{2}\right)-1}(n-\xi)^{q-1} a_{n}^{q}=J^{p}=I \\
& >\frac{\Gamma(\lambda)}{\alpha^{1 / p} \Gamma(\lambda+m)}\left(k_{\lambda}\left(\lambda_{2}\right)\right)^{\frac{1}{p}}\left(k_{\lambda}\left(\lambda_{1}\right)\right)^{\frac{1}{q}}\left[\int_{0}^{\infty} x^{p\left(1-\hat{\lambda}_{1}\right)-1}\left(f^{(m)}(x)\right) d x\right]^{\frac{1}{p}} J^{p-1}>0, \\
J & =\left\{\sum_{n=2}^{\infty}[\ln (n-\xi)]^{q\left(1-\hat{\lambda}_{2}\right)-1}(n-\xi)^{q-1} a_{n}^{q}\right\}^{\frac{1}{p}} \\
& >\frac{\Gamma(\lambda)}{\alpha^{1 / p} \Gamma(\lambda+m)}\left(k_{\lambda}\left(\lambda_{2}\right)\right)^{\frac{1}{p}}\left(k_{\lambda}\left(\lambda_{1}\right)\right)^{\frac{1}{q}}\left[\int_{0}^{\infty} x^{p\left(1-\hat{\lambda}_{1}\right)-1}\left(f^{(m)}(x)\right) d x\right]^{\frac{1}{p}},
\end{aligned}
$$

namely, (17) follows, which is equivalent to (12).

The theorem is proved.

Theorem 4 For $p<0(0<q<1)$, if $\lambda_{1}+\lambda_{2}=\lambda$, then the constant factor

$$
\frac{\Gamma(\lambda)}{\alpha^{1 / p} \Gamma(\lambda+m)}\left(k_{\lambda}\left(\lambda_{2}\right)\right)^{\frac{1}{p}}\left(k_{\lambda}\left(\lambda_{1}\right)\right)^{\frac{1}{q}}
$$

in (17) is best possible. On the other hand, if we add the condition that $\lambda-\lambda_{1} \leq \frac{1}{\alpha}$, the same constant factor in (17) is best possible, then we have $\lambda_{1}+\lambda_{2}=\lambda$.

Proof If $\lambda_{1}+\lambda_{2}=\lambda$, then by Theorem 2 , the constant factor

$$
\frac{\Gamma(\lambda)}{\alpha^{1 / p} \Gamma(\lambda+m)}\left(k_{\lambda}\left(\lambda_{2}\right)\right)^{\frac{1}{p}}\left(k_{\lambda}\left(\lambda_{1}\right)\right)^{\frac{1}{q}}
$$

in (12) is best possible. By (19), the constant factor in (17) is still best possible. Otherwise, we would reach a contradiction that the constant factor in (12) is not best possible.

On the other hand, if the constant factor in (17) is best possible, then, by the equivalency of (17) and (12), in view of $J^{p}=I$ (see the proof of Theorem 3), we still can show that the constant factor in (12) is best possible. By the assumption and Theorem 2, we have $\lambda_{1}+\lambda_{2}=\lambda$.

The theorem is proved.

Remark 1 (i) For $\alpha=1$ in (13) and (18), we have the following equivalent inequalities:

$$
\begin{aligned}
\int_{0}^{\infty} & \sum_{n=2}^{\infty} \frac{a_{n}}{\ln ^{\lambda+m} e^{x}(n-\xi)} f(x) d x \\
> & \frac{\Gamma(\lambda)}{\Gamma(\lambda+m)} B\left(\lambda_{1}, \lambda_{2}\right) \\
& \times\left[\int_{0}^{\infty} x^{p\left(1-\lambda_{1}\right)-1}\left(f^{(m)}(x)\right)^{p} d x\right]^{\frac{1}{p}}\left\{\sum_{n=2}^{\infty}[\ln (n-\xi)]^{q\left(1-\lambda_{2}\right)-1}(n-\xi)^{q-1} a_{n}^{q}\right\}^{\frac{1}{q}},
\end{aligned}
$$




$$
\begin{gathered}
\left\{\sum_{n=2}^{\infty}[\ln (n-\xi)]^{p \lambda_{2}-1}(n-\xi)^{-1}\left[\int_{0}^{\infty} \frac{f(x)}{\ln ^{\lambda+m} e^{x}(n-\xi)} d x\right]^{p}\right\}^{\frac{1}{p}} \\
>\frac{\Gamma(\lambda)}{\Gamma(\lambda+m)} B\left(\lambda_{1}, \lambda_{2}\right)\left[\int_{0}^{\infty} x^{p\left(1-\lambda_{1}\right)-1}\left(f^{(m)}(x)\right)^{p} d x\right]^{\frac{1}{p}} .
\end{gathered}
$$

(ii) For $\xi=0$ in (13) and (18), we have the following equivalent inequalities:

$$
\begin{aligned}
& \int_{0}^{\infty} \sum_{n=2}^{\infty} \frac{a_{n}}{\left(x+\ln ^{\alpha} n\right)^{\lambda+m}} f(x) d x \\
& >\frac{\Gamma(\lambda)}{\alpha^{1 / p} \Gamma(\lambda+m)} B\left(\lambda_{1}, \lambda_{2}\right) \\
& \quad \times\left[\int_{0}^{\infty} x^{p\left(1-\lambda_{1}\right)-1}\left(f^{(m)}(x)\right)^{p} d x\right]^{\frac{1}{p}}\left[\sum_{n=2}^{\infty}(\ln n)^{q\left(1-\alpha \lambda_{2}\right)-1} n^{q-1} a_{n}^{q}\right]^{\frac{1}{q}}, \\
& \left\{\sum_{n=2}^{\infty} \frac{(\ln n)^{p \alpha \lambda_{2}-1}}{n}\left[\int_{0}^{\infty} \frac{f(x)}{\left(x+\ln ^{\alpha} n\right)^{\lambda+m}} d x\right]^{p}\right\}^{\frac{1}{p}} \\
& >\frac{\Gamma(\lambda)}{\alpha^{1 / p} \Gamma(\lambda+m)} B\left(\lambda_{1}, \lambda_{2}\right)\left[\int_{0}^{\infty} x^{p\left(1-\lambda_{1}\right)-1}\left(f^{(m)}(x)\right)^{p} d x\right]^{\frac{1}{p}} .
\end{aligned}
$$

Hence,(13) (resp. (18)) is a more accurate form of (22) (resp. (23)).

(ii) For $\xi=\frac{1}{2}$ in (13) and (18), we have the following equivalent inequalities:

$$
\begin{aligned}
\int_{0}^{\infty} \sum_{n=2}^{\infty} \frac{a_{n}}{\left[x+\ln ^{\alpha}\left(n-\frac{1}{2}\right)\right]^{\lambda+m}} f(x) d x \\
>\frac{\Gamma(\lambda)}{\alpha^{1 / p} \Gamma(\lambda+m)} B\left(\lambda_{1}, \lambda_{2}\right) \\
\quad \times\left[\int_{0}^{\infty} x^{p\left(1-\lambda_{1}\right)-1}\left(f^{(m)}(x)\right)^{p} d x\right]^{\frac{1}{p}} \\
\quad \times\left\{\sum_{n=2}^{\infty}\left[\ln \left(n-\frac{1}{2}\right)\right]^{q\left(1-\alpha \lambda_{2}\right)-1}\left(n-\frac{1}{2}\right){ }^{q-1} a_{n}^{q}\right\}^{\frac{1}{q}} \\
\left\{\sum_{n=2}^{\infty}\left[\ln \left(n-\frac{1}{2}\right)\right]^{p \alpha \lambda_{2}-1}\left(n-\frac{1}{2}\right)^{-1}\left[\int_{0}^{\infty} \frac{f(x)}{\left[x+\ln ^{\alpha}\left(n-\frac{1}{2}\right)\right]^{\lambda+m}} d x\right]^{p}\right\}^{\frac{1}{p}} \\
>\frac{\Gamma(\lambda)}{\alpha^{1 / p} \Gamma(\lambda+m)} B\left(\lambda_{1}, \lambda_{2}\right)\left[\int_{0}^{\infty} x^{p\left(1-\lambda_{1}\right)-1}\left(f^{(m)}(x)\right)^{p} d x\right]^{\frac{1}{p}} .
\end{aligned}
$$

The constant factors in the above inequalities are all best possible.

\section{Another kind of reverses}

Similar to Lemma 2, we have the following. 
Lemma 4 For $0<p<1(q<0)$, we have the following reverse Mulholland-type inequality:

$$
\begin{aligned}
I_{0}= & \int_{0}^{\infty} \sum_{n=2}^{\infty} \frac{a_{n}}{\left[x+\ln ^{\alpha}(n-\xi)\right]^{\lambda}} f^{(m)}(x) d x>\left(\frac{1}{\alpha} k_{\lambda}\left(\lambda_{2}\right)\right)^{\frac{1}{p}}\left(k_{\lambda}\left(\lambda_{1}\right)\right)^{\frac{1}{q}} \\
& \times\left\{\int_{0}^{\infty}\left[1-O\left(\frac{1}{x^{\lambda_{2}}}\right)\right] x^{p\left(1-\hat{\lambda}_{1}\right)-1}\left(f^{(m)}(x)\right)^{p} d x\right\}^{\frac{1}{p}} \\
& \times\left\{\sum_{n=2}^{\infty}[\ln (n-\xi)]^{q\left(1-\alpha \hat{\lambda}_{2}\right)-1}(n-\xi)^{q-1} a_{n}^{q}\right\}^{\frac{1}{q}} .
\end{aligned}
$$

Theorem 5 For $0<p<1(q<0)$, we have the following equivalent reverse inequalities:

$$
\begin{aligned}
I= & \int_{0}^{\infty} \sum_{n=2}^{\infty} \frac{a_{n}}{\left[x+\ln ^{\alpha}(n-\xi)\right]^{\lambda+m}} f(x) d x>\frac{\Gamma(\lambda)}{\Gamma(\lambda+m)}\left(\frac{1}{\alpha} k_{\lambda}\left(\lambda_{2}\right)\right)^{\frac{1}{p}}\left(k_{\lambda}\left(\lambda_{1}\right)\right)^{\frac{1}{q}} \\
& \times\left[\int_{0}^{\infty}\left(1-O\left(\frac{1}{x^{\lambda_{2}}}\right)\right) x^{p\left(1-\overleftarrow{\lambda_{1}}\right)-1}\left(f^{(m)}(x)\right)^{p} d x\right]^{\frac{1}{p}} \\
& \times\left\{\sum_{n=2}^{\infty}[\ln (n-\xi)]^{q\left(1-\alpha \overleftarrow{\lambda_{2}}\right)-1}(n-\xi)^{q-1} a_{n}^{q}\right\}^{\frac{1}{q}} \\
J= & \left\{\sum_{n=2}^{\infty}[\ln (n-\xi)]^{p \alpha \hat{\lambda}_{2}-1}(n-\xi)^{-1}\left[\int_{0}^{\infty} \frac{f(x)}{\left[x+\ln ^{\alpha}(n-\xi)\right]^{\lambda+m}} d x\right]^{p}\right\}^{\frac{1}{p}} \\
> & \frac{\Gamma(\lambda)}{\alpha^{1 / p} \Gamma(\lambda+m)}\left(k_{\lambda}\left(\lambda_{2}\right)\right)^{\frac{1}{p}}\left(k_{\lambda}\left(\lambda_{1}\right)\right)^{\frac{1}{q}} \\
& \times\left[\int_{0}^{\infty}\left(1-O\left(\frac{1}{x^{\lambda_{2}}}\right)\right) x^{p\left(1-\hat{\lambda}_{1}\right)-1}\left(f^{(m)}(x)\right)^{p} d x\right]^{\frac{1}{p}}
\end{aligned}
$$

In particular, for $\lambda_{1}+\lambda_{2}=\lambda$, we have the following equivalent reverse inequalities:

$$
\begin{aligned}
& \int_{0}^{\infty} \sum_{n=2}^{\infty} \frac{a_{n}}{\left[x+\ln ^{\alpha}(n-\xi)\right]^{\lambda+m}} f(x) d x \\
&> \frac{\Gamma(\lambda)}{\alpha^{1 p} \Gamma(\lambda+m)} B\left(\lambda_{1}, \lambda_{2}\right) \\
& \times\left[\int_{0}^{\infty}\left(1-O\left(\frac{1}{x^{\lambda_{2}}}\right)\right) x^{p\left(1-\lambda_{1}\right)-1}\left(f^{(m)}(x)\right)^{p} d x\right]^{\frac{1}{p}} \\
& \times\left\{\sum_{n=2}^{\infty}[\ln (n-\xi)]^{q\left(1-\alpha \lambda_{2}\right)-1}(n-\xi)^{q-1} a_{n}^{q}\right\}^{\frac{1}{q}}, \\
&\left\{\sum_{n=2}^{\infty}[\ln (n-\xi)]^{p \alpha \lambda_{2}-1}(n-\xi)^{-1}\left[\int_{0}^{\infty} \frac{f(x)}{\left[x+\ln ^{\alpha}(n-\xi)\right]^{\lambda+m}} d x\right]^{p}\right\}^{\frac{1}{p}} \\
&> \frac{\Gamma(\lambda)}{\alpha^{1 / p} \Gamma(\lambda+m)} B\left(\lambda_{1}, \lambda_{2}\right)\left[\int_{0}^{\infty}\left(1-O\left(\frac{1}{x^{\lambda_{2}}}\right)\right) x^{p\left(1-\lambda_{1}\right)-1}\left(f^{(m)}(x)\right)^{p} d x\right]^{\frac{1}{p}},
\end{aligned}
$$

where the constant factor $\frac{\Gamma(\lambda)}{\alpha^{1 p} \Gamma(\lambda+m)} B\left(\lambda_{1}, \lambda_{2}\right)$ is best possible. 
Proof We only prove that the constant factor in (29) is best possible. The others are omitted.

For any $0<\varepsilon<p \lambda_{1}$, we set

$$
\begin{aligned}
& \tilde{f}^{(0)}(x)=\tilde{f}(x):=\left\{\begin{array}{ll}
0, & 0<x<1, \\
x^{\lambda_{1}+m-\frac{\varepsilon}{p}-1}, & x \geq 1
\end{array},\right. \\
& \tilde{a}_{n}:=[\ln (n-\xi)]^{\alpha\left(\lambda_{2}-\frac{\varepsilon}{q}\right)-1}(n-\xi)^{-1} \quad(n \in \mathrm{N} \backslash\{1\}),
\end{aligned}
$$

and find

$$
\tilde{f}^{(k)}(x)=\left\{\begin{array}{l}
0, \quad 0<x<1, \\
\prod_{i=0}^{k-1}\left(\lambda_{1}+i-\frac{\varepsilon}{p}\right) x^{\lambda_{1}+m-k-\frac{\varepsilon}{p}-1}, \quad x>1
\end{array} \quad(k=0, \ldots, m)\right.
$$

satisfying $\tilde{f}^{(k)}(0+)=0, \tilde{f}^{(k)}(x)=o\left(e^{t x}\right)(t>0, x \rightarrow \infty ; k=0, \ldots, m-1)$.

If there exists a constant $M\left(\geq \frac{\Gamma(\lambda)}{\alpha^{1 / p} \Gamma(\lambda+m)} B\left(\lambda_{1}, \lambda_{2}\right)\right)$ such that (29) is valid when we replace $\frac{\Gamma(\lambda)}{\alpha^{1 / p} \Gamma(\lambda+m)} B\left(\lambda_{1}, \lambda_{2}\right)$ with $M$, then in particular we have

$$
\begin{aligned}
\tilde{I}= & \int_{0}^{\infty} \sum_{n=2}^{\infty} \frac{\tilde{a}_{n} \tilde{f}(x)}{\left[x+\ln ^{\alpha}(n-\xi)\right]^{\lambda+m}} d x \\
> & M\left\{\int_{0}^{\infty}\left(1-O\left(\frac{1}{x^{\lambda 2}}\right)\right) x^{p\left(1-\lambda_{1}\right)-1}\left(\tilde{f}^{(m)}(x)\right)^{p} d x\right\}^{\frac{1}{p}} . \\
& \times\left\{\sum_{n=2}^{\infty}[\ln (n-\xi)]^{q\left(1-\alpha \lambda_{2}\right)-1}(n-\xi)^{q-1} \tilde{a}_{n}^{q}\right\}^{\frac{1}{q}} .
\end{aligned}
$$

By the decreasingness property of series, we find

$$
\begin{aligned}
\tilde{I}> & M \prod_{i=0}^{m-1}\left(\lambda_{1}+i-\frac{\varepsilon}{p}\right)\left[\int_{1}^{\infty}\left(1-O\left(\frac{1}{x^{\lambda_{2}}}\right)\right) x^{p\left(1-\lambda_{1}\right)-1} x^{p\left(\lambda_{1}-1\right)-\varepsilon} d x\right]^{\frac{1}{p}} \\
& \times\left\{\sum_{n=2}^{\infty}[\ln (n-\xi)]^{q\left(1-\alpha \lambda_{2}\right)-1}(n-\xi)^{q-1}[\ln (n-\xi)]^{q \alpha \lambda_{2}-\alpha \varepsilon-q}(n-\xi)^{-q}\right\}^{\frac{1}{q}} \\
= & M \prod_{i=0}^{m-1}\left(\lambda_{1}+i-\frac{\varepsilon}{p}\right)\left[\int_{1}^{\infty} x^{-\varepsilon-1} d x-\int_{1}^{\infty} O\left(\frac{1}{x^{\lambda_{2}+\varepsilon+1}}\right) d x\right]^{\frac{1}{p}} \\
& \times\left\{[\ln (2-\xi)]^{-\alpha \varepsilon-1}(2-\xi)^{-1}+\sum_{n=3}^{\infty}[\ln (n-\xi)]^{-\alpha \varepsilon-1}(n-\xi)^{-1}\right\}^{\frac{1}{q}} \\
> & M \prod_{i=0}^{m-1}\left(\lambda_{1}+i-\frac{\varepsilon}{p}\right)\left(\frac{1}{\varepsilon}-O(1)\right)^{\frac{1}{p}} \\
& \times\left\{[\ln (2-\xi)]^{-\alpha \varepsilon-1}(2-\xi)^{-1}+\int_{2}^{\infty}[\ln (y-\xi)]^{-\alpha \varepsilon-1}(y-\xi)^{-1} d y\right\}^{\frac{1}{q}}
\end{aligned}
$$




$$
\begin{aligned}
= & \frac{M}{\varepsilon} \prod_{i=0}^{m-1}\left(\lambda_{1}+i-\frac{\varepsilon}{p}\right)(1-\varepsilon O(1))^{\frac{1}{p}} \\
& \times\left\{\varepsilon[\ln (2-\xi)]^{-\alpha \varepsilon-1}(2-\xi)^{-1}+\frac{1}{\alpha}[\ln (2-\xi)]^{-\alpha \varepsilon}\right\}^{\frac{1}{q}} .
\end{aligned}
$$

Replacing $\lambda$ with $\lambda+m$, setting $\tilde{\lambda}_{2}:=\lambda_{2}+\frac{\varepsilon}{p} \in(0, \lambda+m), \tilde{\lambda}_{1}:=\lambda_{1}+m-\frac{\varepsilon}{p} \in(0, \lambda+m)$ in (9), we have

$$
\begin{aligned}
\tilde{I} & =\sum_{n=2}^{\infty}\left\{[\ln (n-\xi)]^{\alpha\left(\lambda_{2}+\frac{\varepsilon}{p}\right)} \int_{1}^{\infty} \frac{x^{\lambda_{1}+m-\frac{\varepsilon}{p}-1}}{\left[x+\ln ^{\alpha}(n-\xi)\right]^{\lambda+m}} d x\right\}[\ln (n-\xi)]^{-\alpha \varepsilon-1}(n-\xi)^{-1} \\
& \leq \sum_{n=2}^{\infty}\left\{[\ln (n-\xi)]^{\alpha \tilde{\lambda}_{2}} \int_{0}^{\infty} \frac{x^{\tilde{\lambda}_{1}-1}}{\left[x+\ln ^{\alpha}(n-\xi)\right]^{\lambda+m}} d x\right\}[\ln (n-\xi)]^{-\alpha \varepsilon-1}(n-\xi)^{-1} \\
& =\sum_{n=2}^{\infty} \omega_{\lambda+m}\left(\tilde{\lambda}_{1}, n\right)[\ln (n-\xi)]^{-\alpha \varepsilon-1}(n-\xi)^{-1} \\
& =k_{\lambda+m}\left(\tilde{\lambda}_{1}\right)\left\{[\ln (2-\xi)]^{-\alpha \varepsilon-1}(2-\xi)^{-1}+\sum_{n=3}^{\infty}[\ln (n-\xi)]^{-\alpha \varepsilon-1}(n-\xi)^{-1}\right\} \\
& <k_{\lambda+m}\left(\tilde{\lambda}_{1}\right)\left\{[\ln (2-\xi)]^{-\alpha \varepsilon-1}(2-\xi)^{-1}+\int_{2}^{\infty}[\ln (y-\xi)]^{-\alpha \varepsilon-1}(y-\xi)^{-1} d y\right\} \\
& =\frac{1}{\varepsilon \alpha} k_{\lambda+m}\left(\tilde{\lambda}_{1}\right)\left\{\varepsilon \alpha[\ln (2-\xi)]^{-\alpha \varepsilon-1}(2-\xi)^{-1}+[\ln (2-\xi)]^{-\alpha \varepsilon}\right\} .
\end{aligned}
$$

Based on the above results, we have

$$
\begin{aligned}
& \frac{1}{\alpha} k_{\lambda+m}\left(\lambda_{1}+m-\frac{\varepsilon}{p}\right)\left\{\varepsilon \alpha[\ln (2-\xi)]^{-\alpha \varepsilon-1}(2-\xi)^{-1}+[\ln (2-\xi)]^{-\alpha \varepsilon}\right\} \\
& \quad>\varepsilon \tilde{I} \\
& \quad>M \prod_{i=0}^{m-1}\left(\lambda_{1}+i-\frac{\varepsilon}{p}\right)(1-\varepsilon O(1))^{\frac{1}{p}}\left\{\varepsilon[\ln (2-\xi)]^{-\alpha \varepsilon-1}(2-\xi)^{-1}+\frac{1}{\alpha}[\ln (2-\xi)]^{-\alpha \varepsilon}\right\}^{\frac{1}{q}} .
\end{aligned}
$$

For $\varepsilon \rightarrow 0^{+}$, in view of the continuity of the beta function, it follows that

$$
\frac{\Gamma(\lambda)}{\alpha^{1 / p} \Gamma(\lambda+m)} B\left(\lambda_{1}, \lambda_{2}\right)=\frac{\Gamma\left(\lambda_{1}\right) \Gamma\left(\lambda_{2}\right)}{\alpha^{1 / p} \Gamma(\lambda+m)}=\frac{B\left(\lambda_{1}+m, \lambda_{2}\right)}{\alpha^{1 / p} \prod_{i=0}^{m-1}\left(\lambda_{1}+i\right)} \geq M
$$

Hence, $M=\frac{\Gamma(\lambda)}{\alpha^{1 / p} \Gamma(\lambda+m)} B\left(\lambda_{1}, \lambda_{2}\right)$ is the best possible constant factor in (29).

The theorem is proved.

In the same way of proving Theorem 4, we have the following.

Theorem 6 For $0<p<1(q<0)$, if we add the condition that $\lambda-\lambda_{1} \leq \frac{1}{\alpha}$, the constant factor

$$
\frac{\Gamma(\lambda)}{\Gamma(\lambda+m)}\left(\frac{1}{\alpha} k_{\lambda}\left(\lambda_{2}\right)\right)^{\frac{1}{p}}\left(k_{\lambda}\left(\lambda_{1}\right)\right)^{\frac{1}{q}}
$$

in (27) (or (28)) is the best possible, then we have $\lambda_{1}+\lambda_{2}=\lambda$. 


\section{Conclusions}

In this paper, following the way of $[4,21]$, by means of the weight functions, HermiteHadamard's inequality, and the techniques of real analysis, a new more accurate reverse half-discrete Mulholland-type inequality with the kernel as $\frac{1}{\left[x+\ln ^{\alpha}(n-\xi)\right]^{\lambda+m}}$ involving one higher-order derivative function is given (for $p<0,0<q<1$ ) in Theorem 1 . The equivalent statements of the best possible constant factor related to a few parameters, the equivalent forms, and several particular inequalities are provided in Theorems 2-4 and Remark 1. Another kind of the reverses is also considered (for $0<p<1, q<0$ ) in Theorems 5-6. The lemmas and theorems provide an extensive account of this type of inequalities.

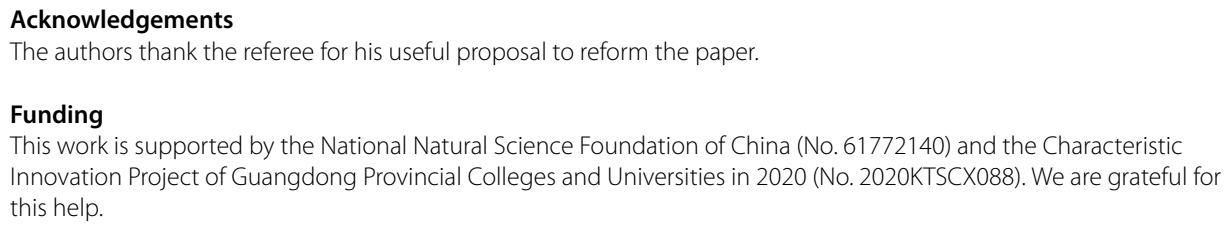

'Department of Computer Science, Guangdong University of Education, Guangzhou, Guangdong 510303, P.R. China.

${ }^{2}$ Department of Mathematics, Guangdong University of Education, Guangzhou, Guangdong 510303, P.R. China.

\section{Publisher's Note}

Springer Nature remains neutral with regard to jurisdictional claims in published maps and institutional affiliations.

Received: 28 June 2021 Accepted: 28 July 2021 Published online: 09 August 2021

References

1. Hardy, G.H., Littlewood, J.E., Polya, G.: Inequalities. Cambridge University Press, Cambridge (1934)

2. Krnić, M., Pečarić, J.: Extension of Hilbert's inequality. J. Math. Anal. Appl. 324(1), 150-160 (2006)

3. Yang, B.C.: On a generalization of Hilbert double series theorem. J. Nanjing Univ. Math. Biq. 18(1), 145-152 (2001)

4. Adiyasuren, V., Batbold, T., Azar, L.E.: A new discrete Hilbert-type inequality involving partial sums. J. Inequal. Appl. 2019, $127(2019)$

5. Yang, B.C.: The Norm of Operator and Hilbert-Type Inequalities. Science Press, Beijing (2009)

6. Krnić, M., Pečarić, J.: General Hilbert's and Hardy's inequalities. Math. Inequal. Appl. 8(1), 29-51 (2005)

7. Perić, l., Vuković, P.: Multiple Hilbert's type inequalities with a homogeneous kernel. Banach J. Math. Anal. 5(2), 33-43 (2011)

8. Huang, Q.L.: A new extension of Hardy-Hilbert-type inequality. J. Inequal. Appl. 2015, 397 (2015)

9. He, B.: A multiple Hilbert-type discrete inequality with a new kernel and best possible constant factor. J. Math. Anal. Appl. 431, 889-902 (2015)

10. Xu, J.S.: Hardy-Hilbert's inequalities with two parameters. Adv. Math. 36(2), 63-76 (2007)

11. Xie, Z.T., Zeng, Z., Sun, Y.F.: A new Hilbert-type inequality with the homogeneous kernel of degree -2. Adv. Appl. Math. Sci. 12(7), 391-401 (2013)

12. Zheng, Z., Gandhi, R.R., Xie, Z.T.: A new Hilbert-type inequality with the homogeneous kernel of degree -2 and with the integral. Bull. Math. Sci. Appl. 3(1), 11-20 (2014)

13. Xin, D.M.: A Hilbert-type integral inequality with the homogeneous kernel of zero degree. Math. Theory Appl. 30(2), 70-74 (2010)

14. Azar, L.E.: The connection between Hilbert and Hardy inequalities. J. Inequal. Appl. 2013, 452 (2013)

15. Adiyasuren, V., Batbold, T., Krnić, M.: Hilbert-type inequalities involving differential operators, the best constants and applications. Math. Inequal. Appl. 18, 111-124 (2015)

16. Rassias, M.T., Yang, B.C.: On half-discrete Hilbert's inequality. Appl. Math. Comput. 220, 75-93 (2013)

17. Yang, B.C., Krnić, M.: A half-discrete Hilbert-type inequality with a general homogeneous kernel of degree 0. J. Math. Inequal. 6(3), 401-417 (2012) 
18. Rassias, M.T., Yang, B.C.: A multidimensional half-discrete Hilbert-type inequality and the Riemann zeta function. Appl. Math. Comput. 225, 263-277 (2013)

19. Rassias, M.T., Yang, B.C.: On a multidimensional half-discrete Hilbert-type inequality related to the hyperbolic cotangent function. Appl. Math. Comput. 242, 800-813 (2013)

20. Yang, B.C., Debnath, L.: Half-Discrete Hilbert-Type Inequalities. World Scientific, Singapore (2014)

21. Hong, Y., Wen, Y.M.: A necessary and sufficient condition of that Hilbert type series inequality with homogeneous kernel has the best constant factor. Ann. Math. 37A(3), 329-336 (2016)

22. Hong, Y:: On the structure character of Hilbert's type integral inequality with homogeneous kernel and application. J. Jilin Univ. Sci. Ed. 55(2), 189-194 (2017)

23. Hong, Y., Huang, Q.L., Yang, B.C., Liao, J.Q.: The necessary and sufficient conditions for the existence of a kind of Hilbert-type multiple integral inequality with the non-homogeneous kernel and its applications. J. Inequal. Appl. 2017, $316(2017)$

24. Xin, D.M., Yang, B.C., Wang, A.Z.: Equivalent property of a Hilbert-type integral inequality related to the beta function in the whole plane. J. Funct. Spaces 2018, Article ID 2691816 (2018)

25. Hong, Y., He, B., Yang, B.C.: Necessary and sufficient conditions for the validity of Hilbert type integral inequalities with a class of quasi-homogeneous kernels and its application in operator theory. J. Math. Inequal. 12(3), 777-788 (2018)

26. Huang, Z.X., Yang, B.C.: Equivalent property of a half-discrete Hilbert's inequality with parameters. J. Inequal. Appl. 2018, $333(2018)$

27. Yang, B.C., Wu, S.H., Wang, A.Z.: On a reverse half-discrete Hardy-Hilbert's inequality with parameters. Mathematics 7 , 1054 (2019)

28. Wang, A.Z., Yang, B.C., Chen, Q.: Equivalent properties of a reverse's half-discrete Hilbert's inequality. J. Inequal. Appl. 2019, 279 (2019)

29. Yang, B.C., Wu, S.H., Liao, J.Q.: On a new extended Hardy-Hilbert's inequality with parameters. Mathematics 8, 73 (2020). https://doi.org/10.3390/math8010073

30. Yang, B.C., Wu, S.H., Wang, A.Z:: On a reverse half-discrete Hardy-Hilbert's inequality with parameters. Mathematics 7(11), 1054 (2019)

31. Kuang, J.C.: Applied Inequalities. Shangdong Science \& Technology Press, Jinan (2004)

32. Kuang, J.C.: Real and Functional Analysis (Continuation), vol. 2. Higher Education Press, Beijing (2015)

\section{Submit your manuscript to a SpringerOpen ${ }^{\circ}$ journal and benefit from:}

- Convenient online submission

- Rigorous peer review

- Open access: articles freely available online

- High visibility within the field

- Retaining the copyright to your article

Submit your next manuscript at $\boldsymbol{~ s p r i n g e r o p e n . c o m ~}$ 